\begin{tabular}{|c|l|}
\hline Title & Confined and interface acoustic phonons in a quantum wire \\
\hline Author(s) & Nishiguchi, Norihiko \\
\hline Citation & $\begin{array}{l}\text { Physical Review B, 50(15), 10970-10980 } \\
\text { https://doi.org/10.1103/PhysRevB.50.10970 }\end{array}$ \\
\hline Issue Date & 1994 10-15 \\
\hline Doc URL & http://hdl.handle.net/2115/47089 \\
\hline Rights & ○ 1994 The A merican Physical Society \\
\hline Type & article \\
\hline File Information & PhysRevB.50.10970.pdf \\
\hline
\end{tabular}

Instructions for use 


\title{
Confined and interface acoustic phonons in a quantum wire
}

\author{
Norihiko Nishiguchi \\ Department of Engineering Science, Hokkaido University, Sapporo 060, Japan
}

(Received 26 May 1994)

\begin{abstract}
Confined and interface acoustic phonon modes in a cylindrical quantum wire embedded in another material are analytically investigated based on the elastic continuum model by means of the potential theory. Confined acoustic phonon modes are coupled modes of bulk-longitudinal and transverse acoustic waves, classified into torsional, dilatational, and flexural modes due to the rotational symmetry of the modes. Dispersions of the confined modes have subband structures with finite cutoff frequencies owing to quantization of wave vectors in the lateral direction. The density of confined phonon states becomes, as a result, a staircaselike structure. As for the interface modes, regions of material parameters for the possible existence of interface modes are investigated. We found that the existence of interface modes in a quantum wire-surrounding system becomes more sensitive to the combinations of materials than that for a plane interface.
\end{abstract}

\section{INTRODUCTION}

Since high electron mobility in a quasi-one-dimensional (Q1D) quantum wire system was predicted by Sakaki, ${ }^{1}$ electron transport properties in Q1D systems have attracted considerable attention and have been extensively studied experimentally ${ }^{2,3}$ and theoretically. ${ }^{4-19}$ The high electron mobility of the systems stems from the reduced dimensionality of electron systems owing to lateral confinement of electrons in the quantum wires. Though early studies of electron scattering with phonons assumed the three-dimensional (3D) bulk character of phonons, optical phonons are experimentally ${ }^{20-22}$ and theoretically ${ }^{23-25}$ known to be confined to a quantum wire embedded within another material (e.g., the GaAs/AlAs systems) because the optical phonon dispersions of the quantum wire and surrounding materials do not overlap in frequency. The optical phonons of the surrounding medium are, as a result, excluded from the quantum wire. In addition to the confined modes, optical interface modes exist at the quantum wire surface $\mathrm{e}^{23,24,26}$ and contribute to electron scattering as do the optical confined phonons. ${ }^{13,23-25}$ The contribution of interface modes to electron scattering is comparable to that of the confined modes and dominates for very thin quantum wires. ${ }^{13,23-25}$

In contrast to optical phonons, acoustic phonons have been assumed to have a bulk $3 \mathrm{D}$ character in most studies of electron energy relaxation, although acoustic phonons are also expected to be confined to a quantum wire embedded in another material. ${ }^{27}$ Suppose a GaAs quantum wire surrounded by AlAs. Because the sound velocities of GaAs are smaller than those of AlAs, acoustic waves in the quantum wire impinging on the wire surface at an angle larger than the critical angle given by Snell's law are totally reflected at the interface. Consequently such waves are confined to the quantum wire, in a manner similar to light waves confined in optical fibers. Dispersions of the confined acoustic phonons should have subband structures due to the confinement of phonons in the lateral direction as well as confined optical phonons, leading to electronic conductance fluctuation with the electric field, as is observed in metallic quantum wires deposited on an insulating substrate. ${ }^{3}$ Waves impinging on the interface at angles smaller than the critical angle can be transmitted through the interface, resulting in extended modes.

Another possible acoustic phonon mode bound to a quantum wire is an interface mode referred to as the Stoneley mode. However, the existence of the interface modes is not evident even for a plane interface because the interface modes occur only for certain combinations of material parameters of the two bulk media. Scholte ${ }^{28}$ studied the region of material parameters and found that Stoneley modes exist at a plane interface when the sound velocities of the two bulk media are almost the same. Wendler and Grigoryan ${ }^{27}$ studied acoustic confined and interface modes in a $\mathrm{Ga}_{1-x} \mathrm{Al}_{x} \mathrm{As} / \mathrm{GaAs} / \mathrm{Ga}_{1-x} \mathrm{Al}_{x} \mathrm{As}$ double heterostructure and showed that interface modes do not occur in this system. The absence of interface modes at a plane interface does not immediately mean the absence of interface modes at a quantum wiresurrounding interface of the same materials as in the case of the plane interface because the region of material parameters for the possible existence of interface modes might be modified by the quantum wire structures.

Though acoustic phonon modes bound to a free standing wire structure have already been studied and several kinds of guided and surface modes have been established, ${ }^{29}$ the confined and interface phonon modes in a quantum wire-surrounding system have not yet been examined. The purpose of this paper is to analytically study two kinds of acoustic phonon modes, confined and interface modes, bound to a quantum wire surrounded by another material based on the elastic continuum model by means of the potential theory. The plan of this paper is as follows. In Sec. II acoustic confined modes are investigated. We give a model for a quantum wire surrounded 
by another material and formalism based on the potential theory. Numerically solving the characteristic equation, we obtain the dispersions of the confined modes and derive the corresponding displacements, considering the $\mathrm{GaAs} / \mathrm{AlAs}$ systems. In Sec. III we investigate the region of combinations of material parameters for the existence of interface modes in the same model given in Sec. II. The dependence of the region on longitudinal wave vectors and on the rotational symmetry order of the modes is discussed. The displacements and dispersions of interface modes are obtained for the $\mathrm{W} / \mathrm{Al}$ systems by numerically solving the characteristic equation. A summary and discussion are presented in Sec. IV.

\section{CONFINED MODES}

\section{A. Model and formalism}

We choose a single cylindrical quantum wire with radius $R$ embedded in another material as the model. This is the simplest theoretical case in order to understand acoustic phonon modes in a quantum wire-surrounding system. We take the wire axis as the $z$ direction and change to cylindrical coordinates $(r, \phi, z)$, considering the cylindrical wire structure. Figure 1 shows the geometry of the quantum wire. Assuming isotropic elasticity for the wire and surrounding materials, the displacement vector $\mathbf{u}$ is given in terms of the scalar potential $\phi_{0}$ and the two vector potentials $\Psi_{1}$ and $\Psi_{2}$ by

$$
\mathbf{u}_{\alpha}=\nabla \phi_{\alpha, 0}+\nabla \times \Psi_{\alpha, 1}+\nabla \times \nabla \times \Psi_{\alpha, 2}
$$

where the vector potentials $\boldsymbol{\Psi}_{1}$ and $\boldsymbol{\Psi}_{2}$ are given by

$$
\boldsymbol{\Psi}_{\alpha, j}=\phi_{\alpha, j} \mathbf{e}_{z}
$$

for $j=1$ and 2. The subscript $\alpha$ denotes the quantum wire $(A)$ and the surrounding medium $(B)$ and $\mathbf{e}_{z}$ is the unit vector in the $z$ direction. Substitution of Eqs. (1) and (2) into a wave equation for the isotropic materials yields the following wave equation for the potential functions $\phi_{\alpha, j}$ :

$$
\rho_{\alpha} \frac{\partial^{2} \phi_{\alpha, j}}{\partial t^{2}}=\left[\delta_{j, 0}\left(C_{\alpha, 11}-C_{\alpha, 44}\right)+C_{\alpha, 44}\right] \nabla^{2} \phi_{\alpha, j},
$$

where $\rho_{\alpha}$ and $C_{\alpha, i j}$ denote the mass densities and stiffness constants of the quantum wire and the surrounding materials, respectively.

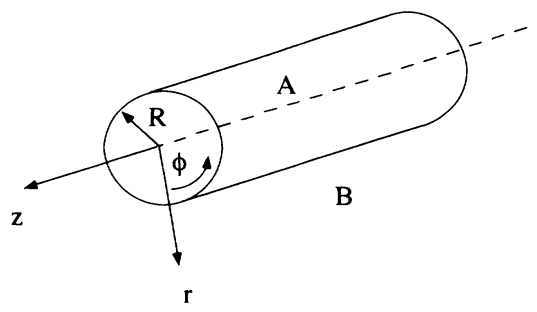

FIG. 1. Geometry of the quantum wire.
Because the stiffness matrix has the same form in cylindrical coordinates as that in Cartesian coordinates, the matrix elements are converted to abbreviated subscript notations by the relations $1=r r, 2=\phi \phi, 3=z z, 4=$ $z \phi, 5=r z$, and $6=r \phi$. Considering the cylindrical symmetry about the wire axis, we may express the potential functions as

$$
\phi_{\alpha, j}(\mathbf{r}, t)=f_{\alpha, j}(r) e^{i(n \phi+q z-\omega t)},
$$

where $n$ is an integer denoting the $n$-fold rotational symmetry of the function, $q$ the wave number in the $z$ direction, and $\omega$ an angular frequency. Inserting Eq. (4) into $(3)$, we obtain the equation for the function $f_{\alpha, j}(r)$ as

$$
\left(\frac{\partial^{2}}{\partial r^{2}}+\frac{1}{r} \frac{\partial}{\partial r}-\frac{n^{2}}{r^{2}}-q^{2}+\frac{\omega^{2}}{v_{\alpha, j}^{2}}\right) f_{\alpha, j}(r)=0,
$$

where $v_{\alpha, j}$ denotes the sound velocities of the bulklongitudinal (LA) and transverse acoustic (TA) waves given by

$$
v_{\alpha, 0}=v_{\alpha, \mathrm{LA}}=\sqrt{\frac{C_{\alpha, 11}}{\rho_{\alpha}}}
$$

and

$$
v_{\alpha, 1}=v_{\alpha, 2}=v_{\alpha, \mathrm{TA}}=\sqrt{\frac{C_{\alpha, 44}}{\rho_{\alpha}}} .
$$

In this section, we consider a GaAs quantum wire buried in AlAs and treat the materials as isotropic media, redefining the stiffness constants $C_{12}$ 's as $C_{12}=$ $C_{11}-2 C_{44}$. Here the stiffness constants and mass density for GaAs are $C_{11}=11.88 \times 10^{11} \mathrm{dyn} \mathrm{cm}^{-2}, C_{44}=$ $5.94 \times 10^{11} \mathrm{dyn} \mathrm{cm}^{-2}$, and $\rho=5.36 \mathrm{~g} \mathrm{~cm}^{-3}$ and for AlAs $C_{11}=12.02 \times 10^{11} \mathrm{dyn} \mathrm{cm}^{-2}, C_{44}=5.89 \times 10^{11} \mathrm{dyn} \mathrm{cm}^{-2}$, and $\rho=3.76 \mathrm{~g} \mathrm{~cm}^{-3}$. The sound velocities of LA and TA waves are

$$
v_{B, \mathrm{LA}}>v_{A, \mathrm{LA}}>v_{B, \mathrm{TA}}>v_{A, \mathrm{TA}}
$$

according to the material parameters.

The wave number $q$ in the longitudinal direction must be smaller than $\omega / v_{A, \mathrm{TA}}$ for the existence of real waves in the quantum wire. Since confined acoustic phonons should be evanescent waves in the surrounding, the longitudinal wave number $q$ must be larger than $\omega / v_{B, \text { TA }}$ to fulfill this condition, due to Snell's law. Thus the longitudinal wave number $q$ must be in the region

$$
\omega / v_{B, \mathrm{TA}}<q<\omega / v_{\mathrm{A}, \mathrm{TA}}
$$

for the existence of the confined acoustic phonon modes.

From these considerations, Eq. (5) is rearranged into a modified Bessel equation for $j=0$ within the quantum wire $(r \leq R)$ as

$$
\left(\frac{\partial^{2}}{\partial r^{2}}+\frac{1}{r} \frac{\partial}{\partial r}-\frac{n^{2}}{r^{2}}-\kappa_{A, 0}^{2}\right) f_{A, 0}(r)=0,
$$

where $\kappa_{A, 0}$ is the wave number in the radial direction given by 


$$
\kappa_{A, 0}=\sqrt{q^{2}-\left(\frac{\omega}{v_{A, \mathrm{LA}}}\right)^{2}}
$$

and the finite solution of Eq. (10) in a quantum wire is given by an associated modified Bessel function of the first kind of the order $n, I_{n}\left(\kappa_{A, 0} r\right)$. The functions $f_{A, j}(r)$ for $j=1$ and 2 in the quantum wire obey the following Bessel equation:

$$
\left(\frac{\partial^{2}}{\partial r^{2}}+\frac{1}{r} \frac{\partial}{\partial r}-\frac{n^{2}}{r^{2}}+k_{A, j}^{2}\right) f_{A, j}(r)=0,
$$

and each finite solution in the quantum wire is given by a Bessel function of the first kind $J_{n}\left(k_{A, j} r\right)$, where the radial wave number $k_{A, j}$ is given by

$$
k_{A, j}=\sqrt{\left(\frac{\omega}{v_{A, \mathrm{TA}}}\right)^{2}-q^{2}}
$$

for $j=1$ and 2 .

In the surrounding medium $(r>R)$, the functions $f_{B, j}(r)$ obey the same modified Bessel equation as Eq. (10), where $\kappa_{A, 0}$ is replaced by $\kappa_{B, j}$, defined by

$$
\kappa_{B, 0}=\sqrt{q^{2}-\left(\frac{\omega}{v_{B, \mathrm{LA}}}\right)^{2}}
$$

and

$$
\kappa_{B, j}=\sqrt{q^{2}-\left(\frac{\omega}{v_{B, \mathrm{TA}}}\right)^{2}}
$$

for $j=1$ and 2, given by an associated Bessel function of the second kind $K_{n}\left(\kappa_{B, j} r\right)$. Thus we obtain the potential functions for a quantum wire

$$
\begin{aligned}
& \phi_{A, 0}(\mathbf{r}, t)=\chi_{A, 0} I_{n}\left(\kappa_{A, 0} r\right) e^{i(n \phi+q z-\omega t)}, \\
& \phi_{A, 1}(\mathbf{r}, t)=\chi_{A, 1} J_{n}\left(k_{A, 1} r\right) e^{i(n \phi+q z-\omega t)}, \\
& \phi_{A, 2}(\mathbf{r}, t)=\frac{\chi_{A, 2}}{k_{A, 2}} J_{n}\left(k_{A, 2} r\right) e^{i(n \phi+q z-\omega t)}
\end{aligned}
$$

and for the surrounding medium

$$
\begin{aligned}
& \phi_{B, 0}(\mathbf{r}, t)=\chi_{B, 0} K_{n}\left(\kappa_{B, 0} r\right) e^{i(n \phi+q z-\omega t)} \\
& \phi_{B, 1}(\mathbf{r}, t)=\chi_{B, 1} K_{n}\left(\kappa_{B, 1} r\right) e^{i(n \phi+q z-\omega t)} \\
& \phi_{B, 2}(\mathbf{r}, t)=\frac{\chi_{B, 2}}{\kappa_{B, 2}} K_{n}\left(\kappa_{B, 2} r\right) e^{i(n \phi+q z-\omega t)}
\end{aligned}
$$

where the $\chi_{\alpha, j}$ 's are coefficients. The coefficients $\chi_{\alpha, j}$ and lateral wave vectors $\kappa_{\alpha, j}$ are determined by the boundary conditions for the displacement and stress fields below. Substituting the potential functions (16) and (17) into Eq. (1), the displacement vector $\mathbf{u}$ is given in vectorial notation $\left(u_{r}, u_{\phi}, u_{z}\right)^{T}$ by

$$
\left[\mathbf{u}_{\alpha}(\mathbf{r}, t)\right]_{i}=\sum_{j}\left[U_{\alpha}(r)\right]_{i j}\left(\mathbf{X}_{\alpha}\right)_{j} e^{i(n \phi+q z-\omega t)}
$$

where

$$
\mathbf{X}_{\alpha}=\left(\begin{array}{c}
\chi_{\alpha, 0} \\
\chi_{\alpha, 1} \\
\chi_{\alpha, 2}
\end{array}\right)
$$

and $U_{\alpha}$ is the matrix given by

$$
U_{A}(r)=\left(\begin{array}{ccc}
\frac{\partial}{\partial r} I_{n}\left(\kappa_{A, 0} r\right) & i \frac{n}{r} J_{n}\left(k_{A, 1} r\right) & i \frac{q}{k_{A, 2}} \frac{\partial}{\partial r} J_{n}\left(k_{A, 2} r\right) \\
i \frac{n}{r} I_{n}\left(\kappa_{A, 0} r\right) & -\frac{\partial}{\partial r} J_{n}\left(k_{A, 1} r\right) & -\frac{n q}{r k_{A, 2}} J_{n}\left(k_{A, 2} r\right) \\
i q I_{n}\left(\kappa_{A, 0} r\right) & 0 & k_{A, 2} J_{n}\left(k_{A, 2} r\right)
\end{array}\right)
$$

for $r \leq R$ and by

$$
U_{B}(r)=\left(\begin{array}{ccc}
\frac{\partial}{\partial r} K_{n}\left(\kappa_{B, 0} r\right) & i \frac{n}{r} K_{n}\left(\kappa_{B, 1} r\right) & i \frac{q}{\kappa_{B, 2}} \frac{\partial}{\partial r} K_{n}\left(\kappa_{B, 2} r\right) \\
i \frac{n}{r} K_{n}\left(\kappa_{B, 0} r\right) & -\frac{\partial}{\partial r} K_{n}\left(\kappa_{B, 1} r\right) & -\frac{n q}{r \kappa_{B, 2}} K_{n}\left(\kappa_{B, 2} r\right) \\
i q K_{n}\left(\kappa_{B, 0} r\right) & 0 & -\kappa_{B, 2} K_{n}\left(\kappa_{B, 2} r\right)
\end{array}\right)
$$

for $r>R$.

The relevant stress vector field $\sigma_{\alpha}\left[=\left(\sigma_{\alpha, r r}, \sigma_{\alpha, r \phi}, \sigma_{\alpha, r z}\right)^{T}\right]$ is explicitly given, by using the displacement vector, as

$$
\left(\begin{array}{c}
\sigma_{\alpha, r r} \\
\sigma_{\alpha, r \phi} \\
\sigma_{\alpha, r z}
\end{array}\right)=\left(\begin{array}{ccc}
C_{\alpha, 11} \frac{\partial}{\partial r}+C_{\alpha, 12} \frac{1}{r} & C_{\alpha, 12} \frac{1}{r} \frac{\partial}{\partial \phi} & C_{\alpha, 12} \frac{\partial}{\partial z} \\
C_{\alpha, 44} \frac{1}{r} \frac{\partial}{\partial \phi} & C_{\alpha, 44}\left(\frac{\partial}{\partial r}-\frac{1}{r}\right) & 0 \\
C_{\alpha, 44} \frac{\partial}{\partial z} & 0 & C_{\alpha, 44} \frac{\partial}{\partial r}
\end{array}\right)\left(\begin{array}{c}
u_{\alpha, r} \\
u_{\alpha, \phi} \\
u_{\alpha, z}
\end{array}\right)
$$


Substituting Eq. (18) into (22), the stress vector is rewritten as

$$
\left[\boldsymbol{\sigma}_{\alpha}(\mathbf{r}, t)\right]_{i}=\sum_{j}\left[T_{\alpha}(r)\right]_{i j}\left(\mathbf{X}_{\alpha}\right)_{j} e^{i(n \phi+q z-\omega t)},
$$

where the matrix elements of $T_{\alpha}$ yield

$$
\begin{aligned}
\left(T_{A}\right)_{11}= & {\left[\left(C_{A, 11}-C_{A, 12}\right) \frac{\partial^{2}}{\partial r^{2}}-C_{A, 12}\left(\frac{\omega}{v_{A, 0}}\right)^{2}\right] } \\
& \times I_{n}\left(\kappa_{A, 0} r\right), \\
\left(T_{A}\right)_{12}= & i n\left(C_{A, 11}-C_{A, 12}\right) \frac{\partial}{\partial r} \frac{J_{n}\left(k_{A, 1} r\right)}{r}, \\
\left(T_{A}\right)_{13}= & i \frac{q}{k_{A, 2}}\left(C_{A, 11}-C_{A, 12}\right) \frac{\partial^{2}}{\partial r^{2}} J_{n}\left(k_{A, 2} r\right), \\
\left(T_{A}\right)_{21}= & 2 i n C_{A, 44} \frac{\partial}{\partial r} \frac{I_{n}\left(\kappa_{A, 0} r\right)}{r} \\
\left(T_{A}\right)_{22}= & -C_{A, 44}\left(2 \frac{\partial^{2}}{\partial r^{2}}+k_{A, 1}^{2}\right) J_{n}\left(k_{A, 1} r\right), \\
\left(T_{A}\right)_{23}= & -2 \frac{n q}{k_{A, 2}} C_{A, 44} \frac{\partial}{\partial r} \frac{J_{n}\left(k_{A, 2} r\right)}{r} \\
\left(T_{A}\right)_{31}= & 2 i q C_{A, 44} \frac{\partial}{\partial r} I_{n}\left(\kappa_{A, 0} r\right), \\
\left(T_{A}\right)_{32}= & -\frac{n q}{r} C_{A, 44} J_{n}\left(k_{A, 1} r\right), \\
\left(T_{A}\right)_{33}= & \left(k_{A, 2}-\frac{q^{2}}{k_{A, 2}}\right) C_{A, 44} \frac{\partial}{\partial r} J_{n}\left(k_{A, 2} r\right)
\end{aligned}
$$

for $r \leq R$ and

$$
\begin{aligned}
\left(T_{B}\right)_{11}= & {\left[\left(C_{B, 11}-C_{B, 12}\right) \frac{\partial^{2}}{\partial r^{2}}-C_{B, 12}\left(\frac{\omega}{v_{B, 0}}\right)^{2}\right] } \\
& \times K_{n}\left(\kappa_{B, 0} r\right) \\
\left(T_{B}\right)_{12}= & i n\left(C_{B, 11}-C_{B, 12}\right) \frac{\partial}{\partial r} \frac{K_{n}\left(\kappa_{B, 1} r\right)}{r}, \\
\left(T_{B}\right)_{13}= & i \frac{q}{\kappa_{B, 2}}\left(C_{B, 11}-C_{B, 12}\right) \frac{\partial^{2}}{\partial r^{2}} K_{n}\left(\kappa_{B, 2} r\right) \\
\left(T_{B}\right)_{21}= & 2 i n C_{B, 44} \frac{\partial}{\partial r} \frac{K_{n}\left(\kappa_{B, 0} r\right)}{r} \\
\left(T_{B}\right)_{22}= & -C_{B, 44}\left(2 \frac{\partial^{2}}{\partial r^{2}}-\kappa_{B, 1}^{2}\right) K_{n}\left(\kappa_{B, 1} r\right) \\
\left(T_{B}\right)_{23}= & -2 \frac{n q}{\kappa_{B, 2}} C_{B, 44} \frac{\partial}{\partial r} \frac{K_{n}\left(\kappa_{B, 2} r\right)}{r} \\
\left(T_{B}\right)_{31}= & 2 i q C_{B, 44} \frac{\partial}{\partial r} K_{n}\left(\kappa_{B, 0} r\right) \\
\left(T_{B}\right)_{32}= & -\frac{n q}{r} C_{B, 44} K_{n}\left(\kappa_{B, 1} r\right) \\
\left(T_{B}\right)_{33}= & -\left(\kappa_{B, 2}+\frac{q^{2}}{\kappa_{B, 2}}\right) C_{B, 44} \frac{\partial}{\partial r} K_{n}\left(\kappa_{B, 2} r\right)
\end{aligned}
$$

for $r>R$. Applying the boundary condition of continuity of the displacement and stress fields at $r=R$ as

$$
\mathbf{u}_{A}(R, \phi, z, t)=\mathbf{u}_{B}(R, \phi, z, t)
$$

and

$$
\sigma_{\boldsymbol{A}}(R, \phi, z, t)=\boldsymbol{\sigma}_{\boldsymbol{B}}(R, \phi, z, t)
$$

we obtain the characteristic equation

$$
\left|U_{A}^{-1} U_{B}-T_{A}^{-1} T_{B}\right|=0,
$$

provided that $\mathbf{X}_{A} \neq 0$ and $\mathbf{X}_{B} \neq 0$.

\section{B. Confined phonon modes and dispersions}

The rotational symmetry order $n$ is a parameter characterizing the confined phonon modes. Hence we obtain the dispersions and displacement vectors by numerically solving the characteristic equation (28) for each rotational symmetry order $n$.

\section{Dispersions}

For $n=0$ there are two azimuthally symmetric modes because $\chi_{1}$ decouples from $\chi_{0}$ and $\chi_{2}$. The mode due to $\nabla \times \Psi_{1}$ has only the azimuthal component $u_{\phi}$. In contrast, the mode due to the sum of terms $\nabla \phi_{0}+\nabla \times \nabla \times \Psi_{2}$ has the radial $u_{r}$ and axial components $u_{z}$ of the displacement vector. The former and latter modes are referred to as the torsional $(T)$ and dilatational $(D)$ modes, respectively, according to particle motion in a solid as shown below. Figures 2(a) and 2(b) illustrate the dispersion curves of the torsional and dilatational modes up to 1 $\mathrm{THz}$ for $R=100 \AA$. Both modes have three dispersion curves quantitatively similar to each other. Every dispersion curve of these modes has a finite cutoff frequency close to the dispersion of the bulk TA mode of AlAs and

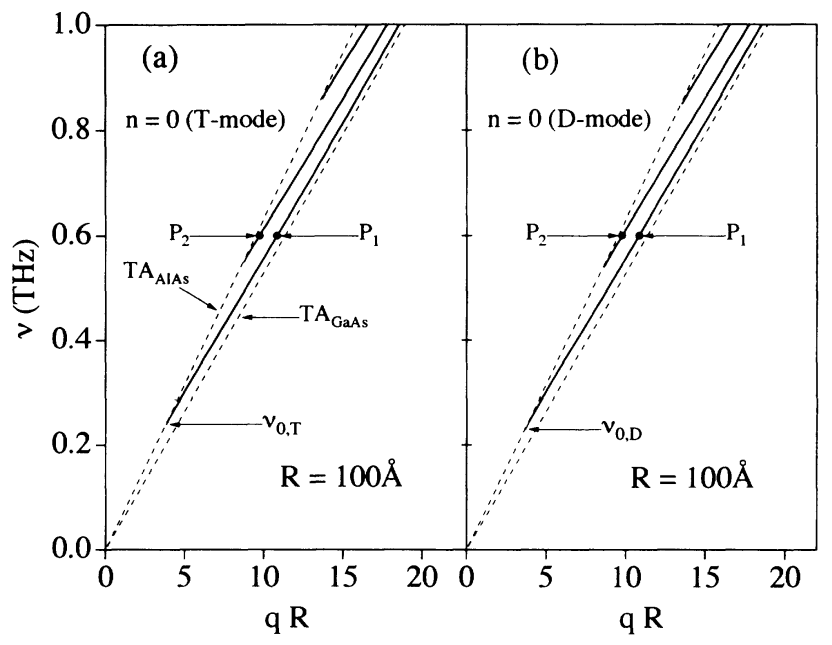

FIG. 2. Dispersions of (a) torsional and (b) dilatational acoustic phonon modes confined to a GaAs quantum wire embedded in AlAs for $R=100 \AA$. There are three dispersion curves (solid lines) with finite cutoff frequencies for both of the modes. The lowest cutoff frequencies $\nu_{0, T}$ and $\nu_{0, D}$ denoted by arrows are almost equal to $0.22 \mathrm{THz}$. The dashed lines are dispersions of the bulk-TA modes of GaAs and AlAs. The points $P_{1}$ and $P_{2}$ at $\nu=0.6 \mathrm{THz}$ are referred to in Figs. 5 and 6. 
tends to that of GaAs with increasing $q$. The lowest cutoff frequencies, $\nu_{0, T}$ of the torsional mode and $\nu_{0, D}$ of the dilatational one, are almost equal to $0.22 \mathrm{THz}$.

For $n \geq 1$, all the terms of Eq. (1) are coupled into one mode termed a flexural mode. The dispersion relations for $n=1$ and 2 are plotted in Figs. 3(a) and 3(b), respectively. There are five dispersion curves with finite cutoff frequencies for both cases. Although the lowest curve is obviously separated from the others, the second and third curves, and the fourth and fifth curves, are close to each other. These phonon subbands shift to a higher frequency region when $n$ increases from 1 to 2 and the lowest cutoff frequency $\nu_{2}$ for $n=2$ becomes larger than $\nu_{1}$ for $n=1$. The differences in frequency between the second and third curves and between the fourth and fifth curves expand with the increase of $n$. These tendencies hold for any positive integers.

The mode with the negative integer $n^{\prime}(=-n)$ has the same dispersions as those of the mode with the positive integer $n$ because the characteristic equation is an even function of $n$. Hence the dispersions of the modes with the same absolute value of $n$ are degenerate.

The phonon subband structures with finite cutoff frequencies lead to staircaselike density of the confined phonon states, as shown in Fig. 4. The lowest cutoff frequency among $\left\{\nu_{n}\right\}$ is $\nu_{1}$ for $n= \pm 1$ and yields $0.12 \mathrm{THz}$ for $R=100 \AA$, below which there is no confined phonon state. This figure also shows the increase of the cutoff frequencies with the rotational symmetry order $|n|$. The density of states tends to the $\nu^{2}$ variation of the bulk $3 \mathrm{D}$

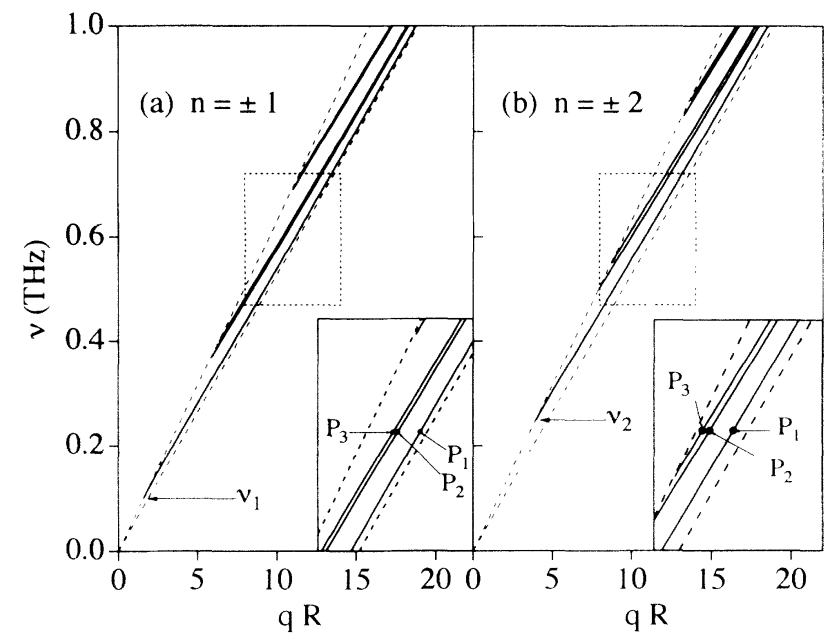

FIG. 3. Dispersions of flexural acoustic phonon modes with (a) $n= \pm 1$ and (b) $n= \pm 2$ confined to a GaAs quantum wire embedded in AlAs for $R=100 \AA$. There are five dispersion curves (solid lines) with finite cutoff frequencies for both of the modes. The lowest cutoff frequencies $\nu_{1}$ and $\nu_{2}$ are denoted by arrows. The second and third dispersion curves and the fourth and fifth ones are close to each other. Insets of (a) and (b) show the regions enclosed by the dotted line. The difference in frequency between the adjacent dispersions expands with increasing $|n|$. The points $P_{1}, P_{2}$, and $P_{3}$ at $0.6 \mathrm{THz}$ are referred to in Figs. 8 and 9. The dashed lines are dispersions of the bulk-TA modes of GaAs and AlAs.

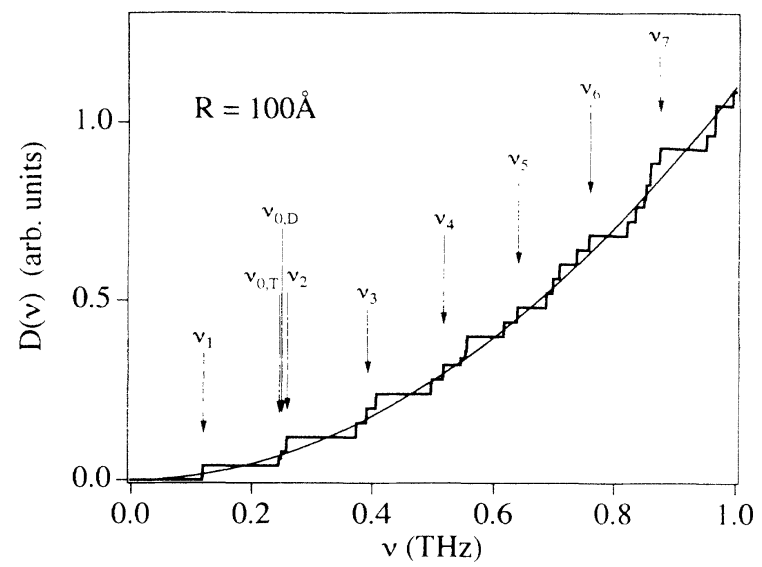

FIG. 4. Density of confined phonon states in the GaAs/AlAs system versus frequency for $R=100 \AA$. The density of confined phonon states shows staircaselike structures owing to the dispersions with finite cutoff frequencies, tending toward the 3D Debye model at high frequencies. The thin solid line denotes the $\nu^{2}$ variation for reference. The increase of the lowest cutoff frequency with $|n|$ for $|n| \geq 1$ is identified in this figure.

Debye model at high frequencies. The thin solid line is drawn to show the $\nu^{2}$ variation for reference.

\section{Displacements}

Figure 5 illustrates amplitude $w_{\phi}$ of the azimuthal displacement of the torsional mode at points $P_{1}$ and $P_{2}$ when $\nu=0.6 \mathrm{THz}$ of the lowest and second dispersions denoted in Fig. 2(a). The amplitude $\mathbf{w}$ is defined by

$$
\mathbf{w}(r)=U_{A}(r) \mathbf{X}_{A} \theta(R-r)+U_{B}(r) \mathbf{X}_{B} \theta(r-R) .
$$

The amplitude $w_{\phi}$ vanishes at $r=0$, varying with increasing $r$ and disappearing at a large $r$. The amplitude belonging to the higher dispersion curve has a node in contrast to the lower one, which shows quantization

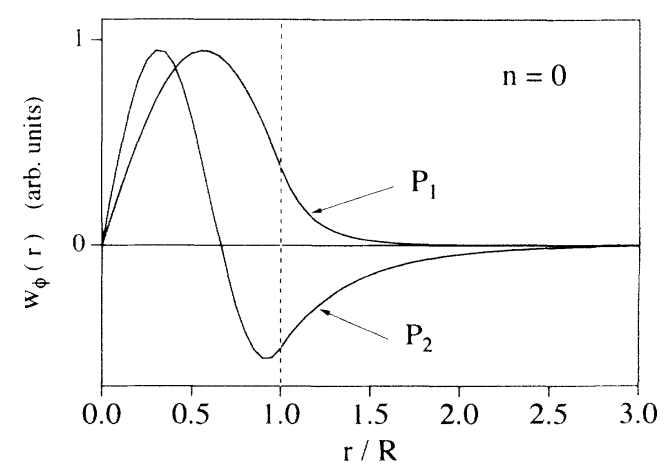

FIG. 5. Amplitudes of the lowest $\left(P_{1}\right)$ and second $\left(P_{2}\right)$ dispersion curves of the torsional modes. The points $P_{1}$ and $P_{2}$ are designated in Fig. 2(a). The dashed line denotes the interface between the wire and the surrounding medium. 
of wave vectors in the lateral direction. The region of phonon confinement of $P_{1}$ is narrower that that of $P_{2}$ because the wave vector $\kappa_{B, 1}$ in the surrounding of $P_{1}$ is larger than that of $P_{2}$ due to the difference in the longitudinal wave vector $q$, as readily understood from Eq. (15).

Figures 6(a) and 6(b) shows the amplitude of the dilatational mode at points $P_{1}$ and $P_{2}$ when $\nu=0.6 \mathrm{THz}$ belonging to the lowest and second dispersions denoted in Fig. 2(b). Only the radial component $w_{r}$ vanishes at $r=0$. Both components $w_{r}$ and $w_{z}$ vary and alternate in sign with increasing $r$, vanishing at a large $r$. The region of phonon confinement of $P_{1}$ is narrower than that of $P_{2}$ for the same reason as with the torsional modes. The number of nodes of each component of $P_{2}$ increases by one in comparison with that of $P_{1}$, showing quantization of wave vectors in the radial direction. There is a phase difference of $\pi / 2$ between $w_{r}$ and $w_{z}$, leading to elliptic particle motion in the plane including the wire axis. The direction of elliptic particle motion depends on the sign of the amplitude. The particle displacement field patterns have the appearance of peristaltic motion of the quantum wire as a whole as shown in Fig. 7.

For finite integers $n$, the phase factors of displacements $u_{\phi}$ and $u_{z}$ are shifted by $\pi / 2$ from $u_{r}$. The phase difference among the components leads to elliptic particle motion oblique to the wire axis. The amplitudes belonging to the lowest three dispersions of the mode with $n=1$ are plotted in Fig. 8. The axial component $w_{z}$ vanishes and the radial component $w_{r}$ equals the azimuthal components $-i w_{\phi}$ at $r=0$. The amplitudes vary with $r$ and rapidly vanish in the surrounding, where the dependence of the region of phonon confinement on $q$ is apparent. These properties hold for all the three curves.

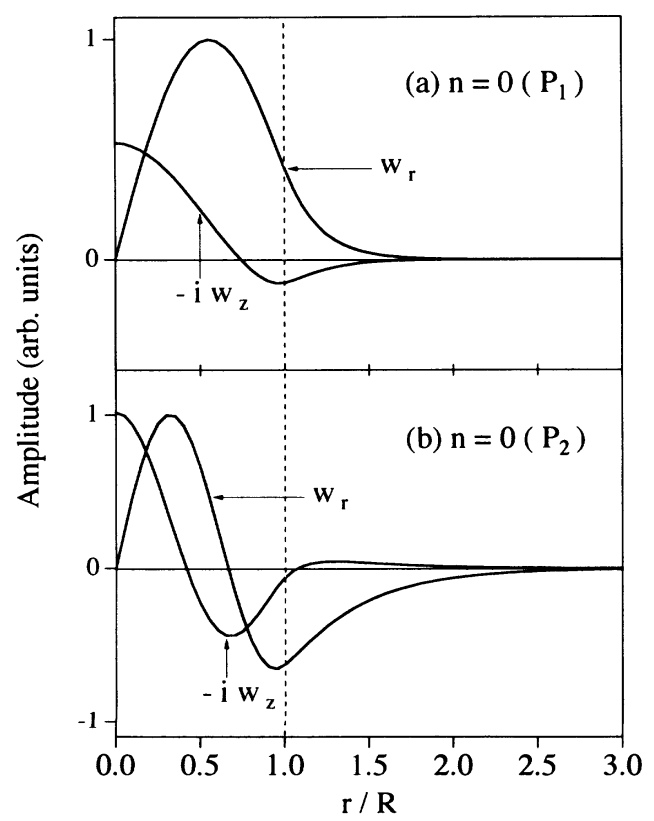

FIG. 6. Amplitudes of (a) the lowest $\left(P_{1}\right)$ and (b) second $\left(P_{2}\right)$ dispersion curves of the dilatational modes. The points $P_{1}$ and $P_{2}$ are designated in Fig. 2(b). The dashed line denotes the interface between the wire and the surrounding medium.

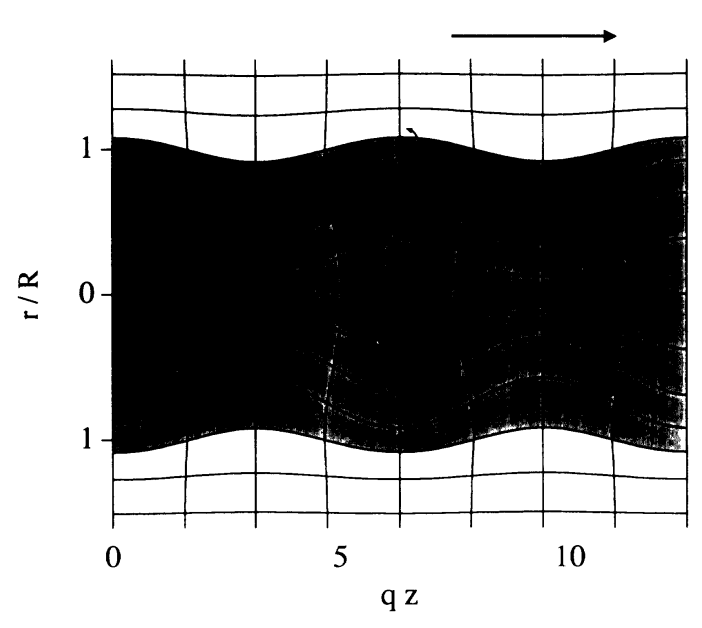

FIG. 7. Displacement field pattern for the dilatational wave traveling in the $z$ direction. The shaded region denotes the wire. The arrows denote the direction of elliptic particle motion with time.

The amplitudes of the lowest dispersion do not have nodes, and the number of nodes increases from low to high dispersion curves, showing quantization of the wave vector in the lateral direction. Aside from the number of nodes, the amplitudes of the lowest and highest dispersions of the three are quite similar because the radial

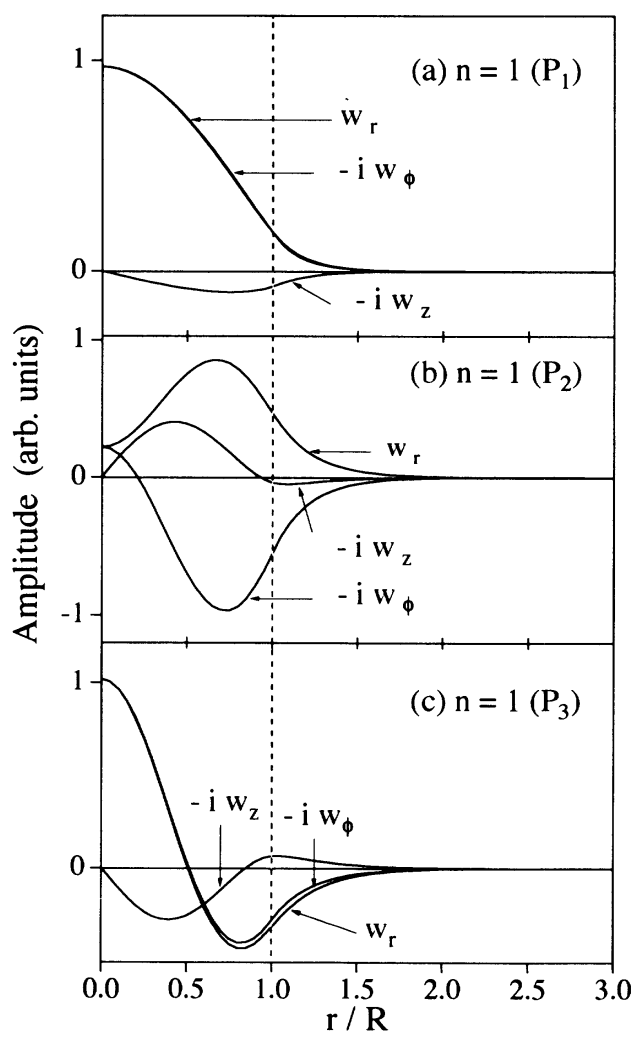

FIG. 8. Amplitudes of (a) the lowest $\left(P_{1}\right),(\mathrm{b})$ second $\left(P_{2}\right)$, and (c) third $\left(P_{3}\right)$ dispersion curves of the flexural mode with $n=1$. The points $P_{1}, P_{2}$, and $P_{3}$ are designated in Fig. 3(a). The dashed line denotes the interface between the wire and the surrounding medium. 
component $w_{r}$ approximately equals $-i w_{\phi}$, independent of $r$. The radial component $w_{r}$ of the second dispersion is almost equal to $i w_{\phi}$, except in the region of the wire axis, in contrast to the other two dispersions. We have studied the amplitudes of higher dispersions and confirmed the relations between the components, i.e., $w_{r} \approx i w_{\phi}$ for the fourth dispersion and $w_{r} \approx-i w_{\phi}$ for the fifth dispersion. Thus the vibrations of modes with $n=1$ are classified into two types owing to the relation between the components $w_{r}$ and $w_{\phi}$.

Figure 9 shows the displacements of the mode with $n=2$ at the points belonging to the lowest three dispersions curves. The properties of amplitudes are the same as those of the mode with $n=1$, except that all the am- plitudes vanish at $r=0$, and there are also two types of vibrations concerning the relation between amplitudes $w_{r}$ and $w_{\phi}$. For a larger rotational symmetry order $n$, the behavior of amplitudes is the same as in the case of $n=2$, including the vanishing of the amplitudes at $r=0$.

The vanishing of amplitudes at $r=0$ is closely related to the rotational symmetry of the modes. Since the displacement vector $\mathbf{u}(\mathbf{r})$ is independent of $\phi$ at $r=0$, the displacements $u_{r}$ of the dilatational modes and $u_{\phi}$ of the torsional modes must vanish at $r=0$ because of the azimuthal symmetry of the modes. Assuming the amplitude $\mathbf{w}$ to be $\left(w_{r}, i w_{\phi}^{\prime \prime}, i w_{z}^{\prime \prime}\right)$, where $w_{r}, w_{\phi}^{\prime \prime}$, and $w_{z}^{\prime \prime}$ are real, the displacement vector $\mathbf{u}$ yields, for a flexural mode with a rotational symmetry order $n$,

$$
\begin{aligned}
\mathbf{u}(\mathbf{r})= & \operatorname{Re}\left[\mathbf{w}(r) e^{i(n \phi+\beta)}\right] \\
= & \frac{w_{r}(r)+w_{\phi}^{\prime \prime}(r)}{2}\{\cos [(n-1) \phi+\beta],-\sin [(n-1) \phi+\beta], 0\} \\
& +\frac{w_{r}(r)-w_{\phi}^{\prime \prime}(r)}{2}\{\cos [(n+1) \phi+\beta], \sin [(n+1) \phi+\beta], 0\}-w_{z}^{\prime \prime}(r)[0,0, \sin (n \phi+\beta)]
\end{aligned}
$$

in Cartesian vectorial notation $\left(u_{x}, u_{y}, u_{z}\right)$, where $\beta=$ $q z-\omega t$. For $n=1$ the $z$ component $w_{z}^{\prime \prime}(0)$ vanishes and $w_{r}(0)$ is required to equal $w_{\phi}^{\prime \prime}(0)$. For $n>1$, all the amplitudes must vanish at $r=0$. These properties of amplitudes at $r=0$ coincide with the results obtained

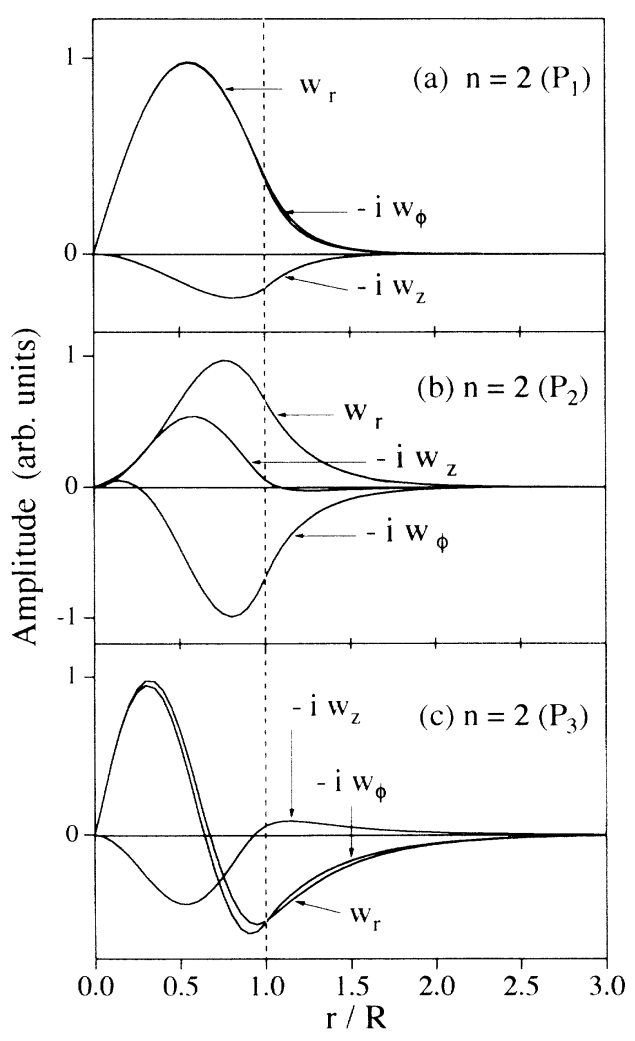

FIG. 9. Amplitudes of (a) the lowest $\left(P_{1}\right),(\mathrm{b})$ second $\left(P_{2}\right)$, and (c) third $\left(P_{3}\right)$ dispersion curves of the flexural mode with $n=2$. The points $P_{1}, P_{2}$, and $P_{3}$ are designated in Fig. 3(b). The dashed line denotes the interface between the wire and the surrounding medium. above and hold universally in the other phonon modes such as interface and extended modes.

Equation (30) is useful to understand the vibrational patterns of the flexural modes. This formula shows that the displacement vector is given by the sum of two kinds of rotations along the wire axis, i.e., clockwise and counterclockwise rotations with angle $\phi$. The amplitudes of the rotations are, interestingly, given by $\left(w_{r}-w_{\phi}^{\prime \prime}\right) / 2$ and $\left(w_{r}+w_{\phi}^{\prime \prime}\right) / 2$, respectively. From these facts, the displacements of the lowest, third, and fifth dispersions of the flexural mode with $n=1$ are found to be independent of angle $\phi$ and the contour of the wire cross section becomes a circle oblique to the wire axis whose center is displaced from the axis. The displacement vector rotates clockwise with increasing $z$ because $\beta=q z-\omega t$. Then the circle oblique to the wire axis rotates along the axis simultaneously with $z$, which results in a spiral rod. The dynamical movement of the wire surface with time looks like a spiral rod rotating counterclockwise with time, which is similar to the motion of a barber pole. The confined acoustic phonon modes with finite integers are thus termed flexural modes according to the motion. In contrast to these cases, the displacement vectors, except for the wire axis region of the second and fourth dispersions, rotate clockwise twice with $\phi$. The whole picture of the quantum wire is also of a spiral rod, but with a deformed cross section because of the $\phi$ dependence of the displacements. The dynamical movement behavior is similar to the former cases.

For $n=2$ the displacements are expressed by the sum of clockwise rotation of a third harmonic and counterclockwise rotation with a period of $\phi$. The displacement vectors belonging to the lowest, third, and fifth dispersions show counterclockwise rotation along the wire axis and the contour of the cross section of the wires becomes a bent oval. The displacement vectors of the second and fourth dispersions show clockwise rotation along the wire axis and the cross section shows twofold rotational symmetry of the modes, although its shape differs from an 
oval. The whole picture of the quantum wire is of a twisted rod with twofold symmetry of the cross section.

Finally, we discuss the displacements for negative $n$ integers. Putting $n=-m$, where $m$ is a positive integer, the displacement vector for a negative integer yields

$$
\begin{aligned}
\mathbf{u}= & \frac{w_{r}+w_{\phi}^{\prime \prime}}{2}\{\cos [(m+1) \phi-\beta], \sin [(m+1) \phi-\beta], 0\} \\
& +\frac{w_{r}-w_{\phi}^{\prime \prime}}{2}\{\cos [(m-1) \phi-\beta], \\
& -\sin [(m-1) \phi-\beta], 0\}+w_{z}^{\prime \prime}[0,0, \sin (m \phi-\beta)] .
\end{aligned}
$$

The displacement vectors belonging to the same dispersion should have the same $\phi$ dependence. Therefore, the azimuthal component $w_{\phi}$ must change its sign in accordance with the sign of the rotational symmetry order $n$. The dynamical movement of the quantum wire shows the barber-pole motion, but its direction changes to clockwise for negative integers because of the change in the sign of $\beta$.

\section{INTERFACE MODES}

As mentioned in Sec. I, the existence of interface modes in a quantum wire depends on combinations of material parameters of the wire and the surrounding media. Thus, in this section, we do not specify the quantum wire and surrounding materials, but investigate combinations of material parameters of the wire and surroundings for the existence of interface modes.

Interface modes should be evanescent waves in the radial direction in both media, for which the longitudinal wave vector $q$ must be larger than $\max \left(\omega / v_{A, \mathrm{TA}}, \omega / v_{B, \mathrm{TA}}\right)$, i.e.,

$$
q>\max \left(\omega / v_{A, \mathrm{TA}}, \omega / v_{B, \mathrm{TA}}\right) \text {. }
$$

All the potential functions in a quantum wire obey modified Bessel equations (10), given by

$$
\begin{aligned}
& \phi_{A, 0}(\mathbf{r}, t)=\chi_{A, 0} I_{n}\left(\kappa_{A, 0} r\right) e^{i(n \phi+q z-\omega t)}, \\
& \phi_{A, 1}(\mathbf{r}, t)=\chi_{A, 1} I_{n}\left(\kappa_{A, 1} r\right) e^{i(n \phi+q z-\omega t)}, \\
& \phi_{A, 2}(\mathbf{r}, t)=\frac{\chi_{A, 2}}{\kappa_{A, 2}} I_{n}\left(\kappa_{A, 2} r\right) e^{i(n \phi+q z-\omega t)},
\end{aligned}
$$

where the radial wave numbers $\kappa_{A, 1}$ and $\kappa_{A, 2}$ are defined by

$$
\kappa_{A, 1}=\kappa_{A, 2}=\sqrt{q^{2}-\left(\frac{\omega}{v_{A, \mathrm{TA}}}\right)^{2}} .
$$

The matrices $U_{A}$ and $T_{A}$ are obtained as follows:

$$
U_{A}(r)=\left(\begin{array}{ccc}
\frac{\partial}{\partial r} I_{n}\left(\kappa_{A, 0} r\right) & i \frac{n}{r} I_{n}\left(\kappa_{A, 1} r\right) & i \frac{q}{\kappa_{A, 2}} \frac{\partial}{\partial r} I_{n}\left(\kappa_{A, 2} r\right) \\
i \frac{n}{r} I_{n}\left(\kappa_{A, 0} r\right) & -\frac{\partial}{\partial r} I_{n}\left(\kappa_{A, 1} r\right) & -\frac{n q}{r \kappa_{A, 2}} I_{n}\left(\kappa_{A, 2} r\right) \\
i q I_{n}\left(\kappa_{A, 0} r\right) & 0 & -\kappa_{A, 2} I_{n}\left(\kappa_{A, 2} r\right)
\end{array}\right)
$$

and

$$
\begin{aligned}
\left(T_{A}\right)_{11}= & {\left[\left(C_{A, 11}-C_{A, 12}\right) \frac{\partial^{2}}{\partial r^{2}}-C_{A, 12}\left(\frac{\omega}{v_{A, 0}}\right)^{2}\right] } \\
& \times I_{n}\left(\kappa_{A, 0} r\right) \\
\left(T_{A}\right)_{12}= & i n\left(C_{A, 11}-C_{A, 12}\right) \frac{\partial}{\partial r} \frac{I_{n}\left(\kappa_{A, 1} r\right)}{r} \\
\left(T_{A}\right)_{13}= & i \frac{q}{\kappa_{A, 2}}\left(C_{A, 11}-C_{A, 12}\right) \frac{\partial^{2}}{\partial r^{2}} I_{n}\left(\kappa_{A, 2} r\right) \\
\left(T_{A}\right)_{21}= & 2 i n C_{A, 44} \frac{\partial}{\partial r} \frac{I_{n}\left(\kappa_{A, 0} r\right)}{r} \\
\left(T_{A}\right)_{22}= & -C_{A, 44}\left(2 \frac{\partial^{2}}{\partial r^{2}}-\kappa_{A, 1}^{2}\right) I_{n}\left(\kappa_{A, 1} r\right), \\
\left(T_{A}\right)_{23}= & -2 \frac{n q}{\kappa_{A, 2}} C_{A, 44} \frac{\partial}{\partial r} \frac{I_{n}\left(\kappa_{A, 2} r\right)}{r} \\
\left(T_{A}\right)_{31}= & 2 i q C_{A, 44} \frac{\partial}{\partial r} I_{n}\left(\kappa_{A, 0} r\right), \\
\left(T_{A}\right)_{32}= & -\frac{n q}{r} C_{A, 44} I_{n}\left(\kappa_{A, 1} r\right), \\
\left(T_{A}\right)_{33}= & -\left(\kappa_{A, 2}+\frac{q^{2}}{\kappa_{A, 2}}\right) C_{A, 44} \frac{\partial}{\partial r} I_{n}\left(\kappa_{A, 2} r\right) .
\end{aligned}
$$

The potentials in the surrounding medium are unchanged, as given by Eq. (17), and then the matrices $U_{B}$ and $T_{B}$ are the same as in Eqs. (21) and (25), respectively. Putting Eqs. (35), (36), (21), and (25) into (28), we obtain the characteristic equation of interface modes. Numerically solving the characteristic equation for each rotational order $n$, we investigate the existence of interface modes at the quantum wire surface.

\section{A. Region of material parameters for the existence of interface modes}

For $n=0$, although $\chi_{1}$ decouples from $\chi_{0}$ and $\chi_{2}$ as well as the confined modes, solutions do not exist for the torsional waves. The reason is straightforward. The displacement of the possible torsional interface mode increases as $I_{1}\left(\kappa_{A, 1} r\right)$ in the quantum wire and decreases as $K_{1}\left(\kappa_{B, 1} r\right)$ in the surrounding medium. Stress becomes positive in the quantum wire and negative in the surrounding, and the discontinuity in stress always occurs at the interface. Thus the boundary condition cannot be fulfilled for the torsional waves.

Figure 10 shows the region of combinations of material 


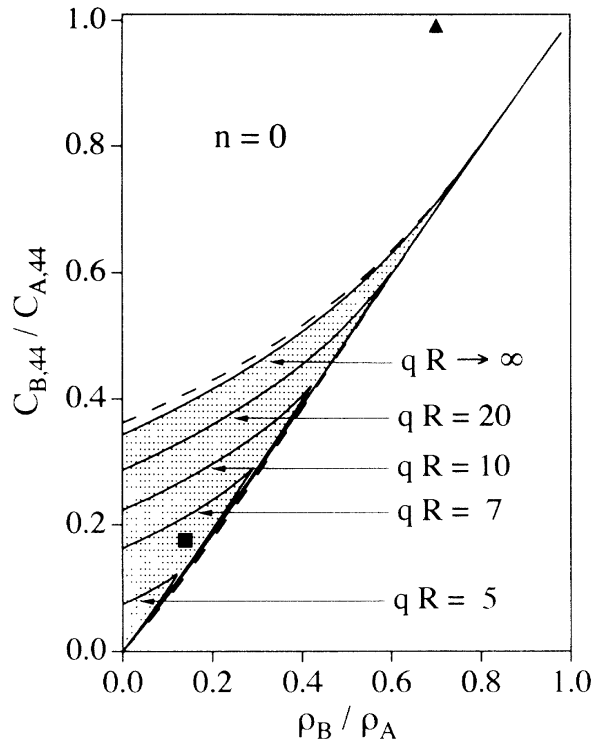

FIG. 10. Range of existence of the dilatational interface mode. The shaded region denotes the region of existence of the interface modes at a plane interface. These results are obtained by assuming $v_{\mathrm{LA}} / v_{\mathrm{TA}}=\sqrt{3}$ for the quantum wire and the surrounding media. The dashed line denotes the contour of the region for the existence of interface modes at a plane interface where $v_{\mathrm{LA}} / v_{\mathrm{TA}}=\sqrt{2}$ is assumed. The solid triangle and square denote the combinations of the $\mathrm{GaAs} / \mathrm{AlAs}\left(\rho_{\mathrm{AlAs}} / \rho_{\mathrm{GaAs}}=0.70\right.$ and $\left.C_{\mathrm{AlAs}, 44} / C_{\mathrm{GaAs}, 44}=0.99\right)$ and $\mathrm{W} / \mathrm{Al}\left(\rho_{\mathrm{Al}} / \rho_{\mathrm{W}}=0.14\right.$ and $\left.C_{\mathrm{Al}, 44} / C_{\mathrm{W}, 44}=0.17\right)$, respectively.

parameters for the existence of a dilatational interface mode together with the region of the interface modes at a plane interface. These results are obtained for the ratio $v_{\mathrm{LA}} / v_{\mathrm{TA}}=\sqrt{3}$ for both media, assuming the stiffness constants $C_{12}=C_{44}$ for them. The shaded region denotes the region for a plane interface, which is a limit of infinite radius for the quantum wire. The regions with a reduced longitudinal wave number $q R$ are enclosed by solid lines. The region contracts with decreasing $q R$. This means that, for a combination of materials, the existence of a dilatational interface mode is limited to a larger $q R$ than a magnitude, at which the combination of materials is located on the contour of the region. In other words, the dispersion of the dilatational interface mode begins at a finite wave number or frequency as will be shown below. The region of the existence of the dilatational interface mode vanishes at the critical reduced longitudinal wave number $q_{\mathrm{cr}} R=4.2$. At smaller than the critical value, no dilatational interface mode exits in any combination of materials. Although Fig. 10 shows the range $C_{B, 44} / C_{A, 44}<1$ and $\rho_{B, 44} / \rho_{A, 44}<1$, there is a region of the existence of the dilatational interface mode in the range $C_{B, 44} / C_{A, 44}>1$ and $\rho_{B, 44} / \rho_{A, 44}>1$. The shrinkage of the region from that of the interface mode at a plane interface with decreasing the reduced wave number $q R$ also occurs.

For a finite integer $n$, there are modes termed flexural interface modes as well as confined modes. Because the characteristic equation is an even function of $n$, the region for a positive integer $n$ is same as that for $-n$. Figure 11 plots the dependence of the region on $n$ at $q R=10$. The region shrinks with increasing $|n|$ for $|n| \geq 1$ and vanishes for $|n|>3$. That is, the existence of the flexural interface modes are limited to $|n| \leq 3$ at $q R=10$. With decreasing $q R$, the region of the flexural interface modes contracts and vanishes as well as the dilatational interface mode. The critical reduced wave number $q_{\mathrm{cr}, n} R$ of the vanishing of the region depends on $n$ and yields $0.1,4.2,7.4,10.1$, and 12.8 for $|n|=1,2,3,4$, and 5, respectively. From these results, the flexural interface modes with $n$ appear when $q R \geq q_{\mathrm{cr}, n} R$ and then the number of the flexural interface modes increases with an increase of $q R$.

Thus the region of material parameters for the possible existence of the interface modes in a quantum wire-surrounding system simply contracts inside the region for a plane interface and does not extend outside the region for a plane interface. In other words, the interface modes of the quantum wire--surrounding systems occur only for a combination of materials which supports the interface modes at a plane interface.

Here we examine the existence of interface modes in the GaAs/AlAs and W/Al systems, which were studied for interface modes in the double heterostructures by Wendler and Grigoryan. ${ }^{27}$ Since the ratios of stiffness constants $C_{\mathrm{AlAs}, 44} / C_{\mathrm{GaAs}, 44}$ and mass densities $\rho_{\mathrm{AlAs}, 44} / \rho_{\mathrm{GaAs}, 44}$ are 0.99 and 0.70 , respectively, the combination of GaAs/AlAs is apart from the region of the possible existence of interface modes at a plane interface, as denoted by a solid triangle in Fig. 10. Then the GaAs/AlAs systems support only the confined phonon modes. In contrast to the GaAs/AlAs systems, the combination of $\mathrm{W} / \mathrm{Al}$ is located within the region for a plane interface (the solid square in Fig. 10); therefore, the sys-

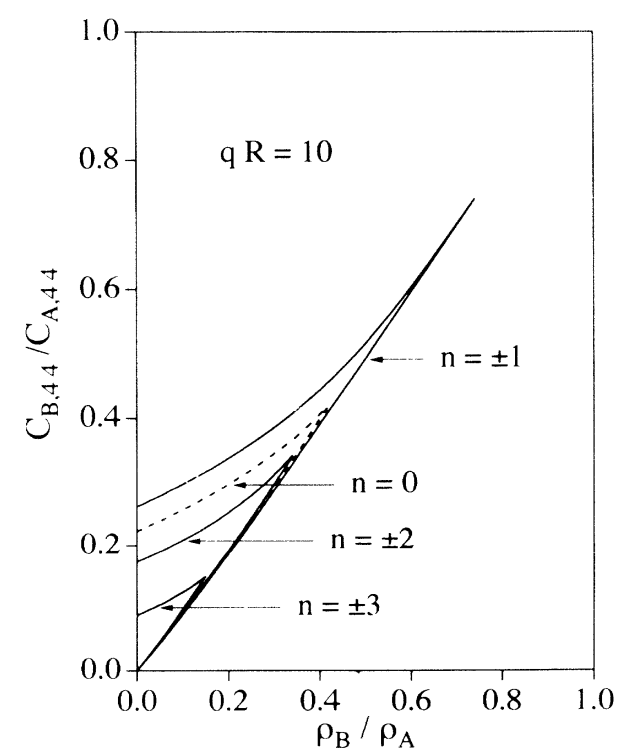

FIG. 11. Dependence of the region of existence of the flexural interface mode on the rotational symmetry order. The dashed line denotes the contour of region for the dilatational mode $(n=0)$ for reference. These results are obtained by assuming $v_{\mathrm{LA}} / v_{\mathrm{TA}}=\sqrt{3}$. 
tems support both interface and confined phonon modes.

It should be noted here that, though the ratio of sound velocities $v_{\mathrm{LA}} / v_{\mathrm{TA}}(\approx \sqrt{2})$ for the $\mathrm{GaAs} / \mathrm{AlAs}$ systems is smaller than the $\sqrt{3}$ assumed in Fig. 10, the regions for the existence of interface modes are almost unchanged due to the difference in the ratios. The dashed line in Fig. 10 denotes the contour of the region for a plane interface at $v_{\mathrm{LA}} / v_{\mathrm{TA}}=\sqrt{2}$.

\section{B. Dispersions and displacement}

The disappearance of the regions with a decrease of the reduced wave number $q R$ and with an increase of the absolute value of $n$ leads to a subband structure with finite cutoff frequencies. Figure 12 shows the dispersion curves of interface modes for the $\mathrm{W} / \mathrm{Al}$ systems. The lowest dispersion curve is the flexural mode with $n= \pm 1$, which begins at a finite longitudinal wave number. The number of interface modes increases with increasing $q R$ and all the dispersion curves tend toward that of the Stoneley modes at a plane interface and should converge on the dispersion for a plane interface at a very large $q R$.

Figure 13(a) illustrates the amplitudes of the dilatational interface mode at $q R=10$. The radial component $w_{r}$ vanishes at $r=0$ due to the azimuthal symmetry of the mode and the axial component $w_{z}$ is almost constant within the wire. There is a phase difference $\pi / 2$ between the components $w_{r}$ and $w_{z}$, leading to elliptic particle motion in the plane including the wire axis. The particle displacement field patterns become peristaltic motion of the quantum wire similar to the dilatational confined modes. The amplitudes of flexural interface modes with $n=1$ at $q R=10$ are plotted in Figs. 13(b). The phase factor of radial component $w_{r}$ is also shifted by $\pi / 2$ from

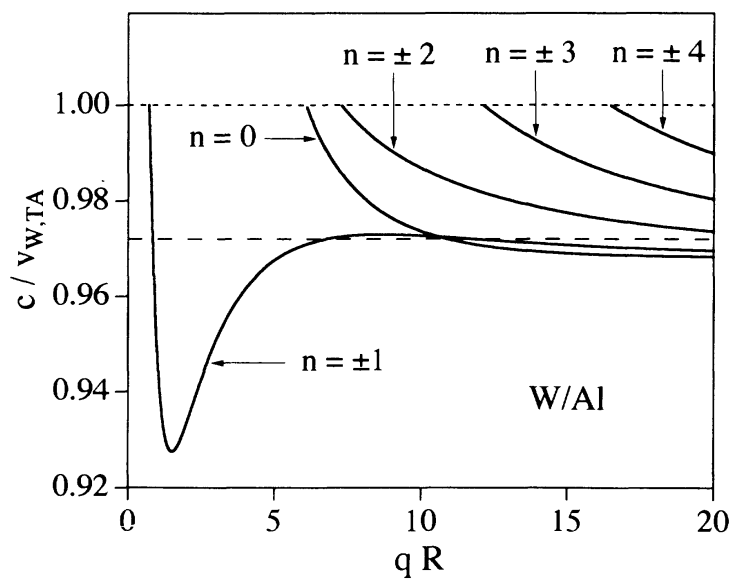

FIG. 12. Phase velocities of the interface modes in the W/Al systems. $c$ denotes the phase velocity of interface modes. The long-dashed line denotes the dispersion of the Stoneley modes at a plane interface. The material parameters used here are $\rho_{\mathrm{W}}=19.317 \mathrm{~g} \mathrm{~cm}^{-3}, C_{\mathrm{W}, 11}$ $=5.233 \times 10^{12} \mathrm{dyn} \mathrm{cm}^{-2}$, and $C_{W, 44}=1.607 \times 10^{12} \mathrm{dyn} \mathrm{cm}^{-2}$ for $\mathrm{W}$ and $\rho_{\mathrm{Al}}=2.733 \mathrm{~g} \mathrm{~cm}^{-3}, C_{\mathrm{Al}, 11}=1.068 \times 10^{12}$ dyn $\mathrm{cm}^{-2}$, and $C_{\mathrm{Al}, 44}=0.282 \times 10^{12} \mathrm{dyn} \mathrm{cm}^{-2}$ for Al.

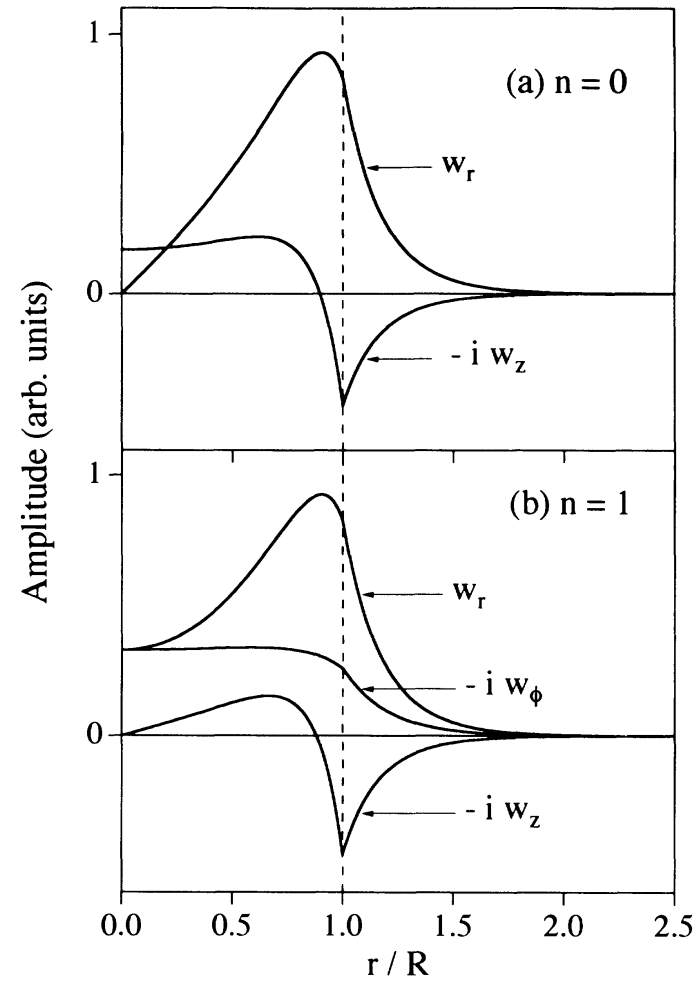

FIG. 13. Amplitudes of (a) dilatational and (b) flexural interface modes in the $\mathrm{W} / \mathrm{Al}$ systems. These results are obtained at $q R=10$. The material parameters used are same as in Fig. 12. The dashed line denotes the interface between the wire and the surrounding medium.

those of $w_{\phi}$ and $w_{z}$ and the displacement vector shows elliptic particle motion in the plane oblique to the wire axis. The radial and azimuthal components $w_{r}$ and $w_{\phi}$ are finite even at the wire axis and comparable to those at the wire surface and there is no relation between the components $w_{r}$ and $w_{\phi}$ as shown for the flexural confined modes. Finally, the amplitudes for $n \geq 2$ are qualitatively similar to those for $n=1$, except for the wire axis region, where all the components vanish at $r=0$ due to the rotational symmetry of the modes.

\section{SUMMARY AND DISCUSSION}

We have investigated confined and interface acoustic phonon modes in a cylindrical quantum wire embedded in another material based on the elastic continuum model by means of the potential theory. These acoustic phonon modes are expressed in terms of a scalar and two vector potentials. The confined modes are classified into three types of modes, i.e., the torsional, dilatational, and flexural modes, due to the rotational symmetry of the modes along the wire axis. The dispersions of the confined modes in the GaAs/AlAs system appear between the bulk TA modes of GaAs and AlAs and have subband structures with finite cutoff frequencies owing to quantization of wave vectors in the lateral direction. The density of confined phonon states appears as a staircase- 
like structure according to the cutoff frequencies, tending toward the $\nu^{2}$ variation of the 3D Debye model with increasing frequency. The phase difference in the amplitudes results in elliptic particle motion of displacement vectors in a plane including the wire axis for the dilatational mode and in a plane oblique to the wire axis for the flexural modes. The dynamical movement of the quantum wire shows peristaltic and barber-pole motion, respectively.

As for the interface modes in the wire--surrounding systems, we examined the regions of possible existence of dilatational and flexural interface modes and found that they shrink inside the region for a plane interface with a decrease in the reduced longitudinal wave vector $q R$ and with an increase in the rotational symmetry order $|n|$. Consequently, the existence of interface modes becomes more sensitive to combinations of material parameters than the case of a plane interface and novel combinations of material parameters specific to the wire-surrounding systems do not appear. Therefore, the interface modes of the quantum wire-surrounding systems are expected only for a combination of materials which has Stoneley modes at a plane interface. The GaAs/AlAs and W/Al systems were examined for the existence of interface modes and we found that the GaAs/AlAs systems support only the confined phonon modes, in contrast to the W/Al systems.

Acoustic phonons are responsible for electron energy relaxation at low temperatures. In Q1D wirelike structures the confined phonon modes should dominate the dissipation processes in the temperature region and electron transport properties will be affected by the phonon subband structures. Recently Senna and Das Sarma ${ }^{14}$ reported enhancement of interaction between electron and acoustic phonons caused by a many-body effect in Q1D systems. As a consequence, peculiar modifications in electron energy relaxation and the related transport phenomena are expected.

\section{ACKNOWLEDGMENTS}

This work was supported in part by a Grant-in-Aid for Scientific Research from the Ministry of Education, Science, and Culture of Japan (Grant No. 05650048).
${ }^{1}$ H. Sakaki, Jpn. J. Appl. Phys. 19, L735 (1980).

${ }^{2}$ J. Cibert, P. M. Petroff, G. J. Dolan, S. J. Pearton, A. C. Gossard, and J. H. Englishman, Appl. Phys. Lett. 49, 1275 (1986).

${ }^{3}$ J. Seyler and M. N. Wybourne, Phys. Rev. Lett. 69, 1427 (1992).

${ }^{4}$ V. K. Arora, Phys. Rev. B 23, 5611 (1981).

${ }^{5}$ G. Fishman, Phys. Rev. B 34, 2394 (1986).

${ }^{6}$ G. Fishman, Phys. Rev. B 36, 7448 (1987).

${ }^{7}$ M. A. Stroscio, Phys. Rev. B 40, 6428 (1989).

${ }^{8}$ U. Bockelmann and G. Bastard, Phys. Rev. B 42, 8947 (1990).

${ }^{9}$ G. Y. Hu and R. F. O'Connel, J. Phys. Condens. Matter 3, 4633 (1991).

${ }^{10}$ P. P. Basu, D. Chattopadhyay, and P. C. Rakshit, Phys. Rev. B 46, 13254 (1992).

${ }^{11}$ V. B. Campos and S. Das Sarma, Phys. Rev. B 46, 3849 (1992).

${ }^{12}$ W. S. Li, S. W. Gu, T. C. Au-Yeung, and Y. Y. Yeung, Phys. Lett. A 166, 377 (1992).

${ }^{13}$ R. Mickevicius, V. V. Mitin, K. W. Kim, M. A. Stroscio, and G. J. Iafrate, J. Phys. Condens. Matter 4, 4959 (1992).

${ }^{14}$ J. R. Senna and S. Das Sarma, Phys. Rev. Lett. 70, 2593 (1993).

${ }^{15}$ S. Das Sarma and V. B. Campos, Phys. Rev. B 47, 3728 (1993).
${ }^{16}$ N. Telang and S. Bandyopadhyay, Appl. Phys. Lett. 62, 3161 (1993).

${ }^{17}$ B. Tanatar, J. Phys. Condens. Matter 5, 2203 (1993).

${ }^{18}$ F. Comas, C. Trallero-Giner, and A. Cantarero, Phys. Rev. B 47, 7602 (1993).

${ }^{19}$ P. A. Knipp and T. L. Reinecke, Phys. Rev. B 48, 5700 (1993).

${ }^{20}$ A. K. Sood, J. Menéndez, M. Cardona, and K. Ploog, Phys. Rev. Lett. 54, 2111 (1985).

${ }^{21}$ G. Fasol, M. Tanaka, H. Sakaki, and Y. Horikoshi, Phys. Rev. B 38, 6056 (1988).

${ }^{22}$ M. Watt, C. M. Sotomayor-Torres, H. E. G. Arnot, and S. P. Beaumont, Semicond. Sci. Technol. 5, 285 (1990).

${ }^{23}$ N. C. Constantinou and B. K. Ridley, Phys. Rev. B 41, 10622 (1990).

${ }^{24}$ R. Enderlein, Phys. Rev. B 47, 2162 (1993).

${ }^{25}$ W. Kim, M. A. Stroscio, A. Bhatt, R. Mickevicius, and V. V. Mitin, J. Appl. Phys. 70, 319 (1991).

${ }^{26}$ P. A. Knipp and T. L. Reinecke, Phys. Rev. B 45, 9091 (1992).

${ }^{27}$ L. Wendler and V. G. Grigoryan, Surf. Sci. 206, 203 (1988).

${ }^{28}$ J. G. Scholte, Mon. Not. R. Astron. Soc. Geophys. Suppl. 5, 120 (1947).

${ }^{29}$ See, for example, B. A. Auld, Acoustic Fields and Waves in Solids (Wiley, New York, 1973), Vol. II, Chap. 10, and references quoted therein. 


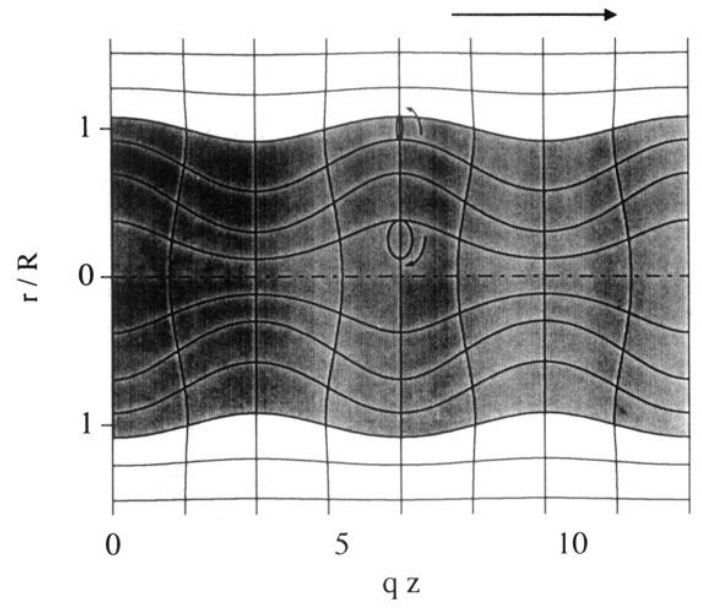

FIG. 7. Displacement field pattern for the dilatational wave traveling in the $z$ direction. The shaded region denotes the wire. The arrows denote the direction of elliptic particle motion with time. 